\title{
Direct Observation of Redox Switching in Resistive Memory Devices Operated In- situ in a Transmission Electron Microscope by Electron Energy Loss Spectroscopy and Off-Axis Electron Holography
}

\author{
David Cooper ${ }^{1,2}$, Nicolas Bernier ${ }^{1,2}$, Christoph Baumer ${ }^{3}$, Rafal Dunin-Borkowski ${ }^{3}$ and Regina Dittmann ${ }^{3}$ \\ 1. Univ. Grenoble Alpes, F-38000 Grenoble, France. \\ 2. CEA, LETI, Minatec Campus, F-38054 Grenoble, France. \\ 3. Peter Grunberg Institute, FZ Julich, D-52425 Julich, Germany.
}

The $\mathrm{Pt} / \mathrm{SrTiO}_{3} / \mathrm{Nb}: \mathrm{SrTiO}_{3}$ system is considered to be a model system for the study of resistive memories [1]. It is thought that changes in the oxygen concentration underneath the top electrode are responsible for the switching. During the past few years, a bewildering array of characterisation studies have appeared in the literature presenting contradictory views about the operation of these resistive memories. It is desirable to improve these devices by correct determination of the switching mechanism rather than using empirical approaches. Transmission electron microscopy (TEM) is a suitable characterisation technique and the use of modern aberration corrected TEMs allow a range of complimentary characterisation techniques to be performed during one experiment on the same specimen. In this presentation we will present experimental results that have been obtained using aberration-corrected scanning (S)TEM, electron energy-loss spectroscopy (EELS) for composition and bonding measurements and off-axis electron holography for measurements of electrostatic potentials that have been obtained on different types of $\mathrm{SrTiO}_{3}$ based memory devices that are switched in-situ in the TEM.

Specimens comprising a Nb:doped $\mathrm{SrTiO}_{3}$ substrate (bottom electrode), a 18-nm-thick Sr-enriched $\mathrm{SrTiO}_{3}$ (Sr:STO) film and a 10-nm-thick Pt (top electrode) with a top Au contact have been examined using a Nanofactory specimen holder comprising a movable piezo-electrical contact. Figure 1(a) shows a low magnification STEM image of one of the devices, the substrate of the device has been electrically connected and the movable probe is placed onto the Au top contact far from the region of interest. Figure 1(b) shows bright-field STEM images of the device that have been acquired before and after switching. Directly after specimen preparation, a regular domain pattern can be seen in the Sr:STO region, however after switching it can be seen that the domains have disappeared in the $4 \mathrm{~nm}$ directly under the Pt electrode. The device was switched several times and EELS spectra were acquired at the low resistance state (LRS) and high resistance state (HRS). Figure 1(c) shows an oxygen map that has been acquired by EELS with the device in a LRS. Figure 1(d) shows profiles that have been extracted from across the devices in both a LRS and HRS. From the oxygen profiles it can be seen that oxygen vacancies $(\mathrm{V} \ddot{)})$ are present in the Sr:STO film and that there is a higher concentration of $\mathrm{Vö}$ at the $\mathrm{Pt}$ electrode interface. These results were found to be reversable and reproducible over several switch cycles. In this presentation we will give more details about the changes in the device during switching and also discuss the size of the "filament". Measurements of the potential measured by off-axis electron holography will also be presented [3].

To investigate the changes in oxygen inside the metal electrode, specimens were grown using a $\mathrm{Rh} / \mathrm{Ti}$ top electrode which is less opaque to electrons in the TEM. Figure 2(a) and (b) show high resolution STEM images that have been acquired of the device before and after switching, re-distribution of the metal in the electrode can be seen. Oxygen maps that have been acquired of the device before switching and in a LRS and HRS are shown in Figure 2(c). The oxygen profiles shown in Figure 2(d) suggest that 
although a large difference can be observed between the virgin and switched specimens, only slight differences can be seen in the LRS and HRS states. However, these results do suggest that the oxygen concentration across the electrode is less abrupt in the LRS and confirm the movement of oxygen in the top electode during switching.

As well as proposing a switching mechanism for these resistive devices based on our in-situ experimental results, we will also present information about the experimental procedure, the reproducibility of the results as well as the significant effects of specimen heating during examination. We will show that by combining careful specimen preparation with state-of-the-art electron microscopes, these types of in-situ measurements that use a movable probe to provide an electrical stimulus can now routinely be used to determine the switching mechanisms in a range of different materials using advanced TEM techniques [4].

\section{References:}

[1] R Waser and M. Aono, Nature Materials 6 (2007) p. 833.

[2] D A Muller et al, Nature 430 (2004), p. 657.

[3] A. Marchewka et al, Sci Reports 4 (2014) 6975.

[4] We acknowledge the European Research Council for funding the Starting Grant "Holoview".
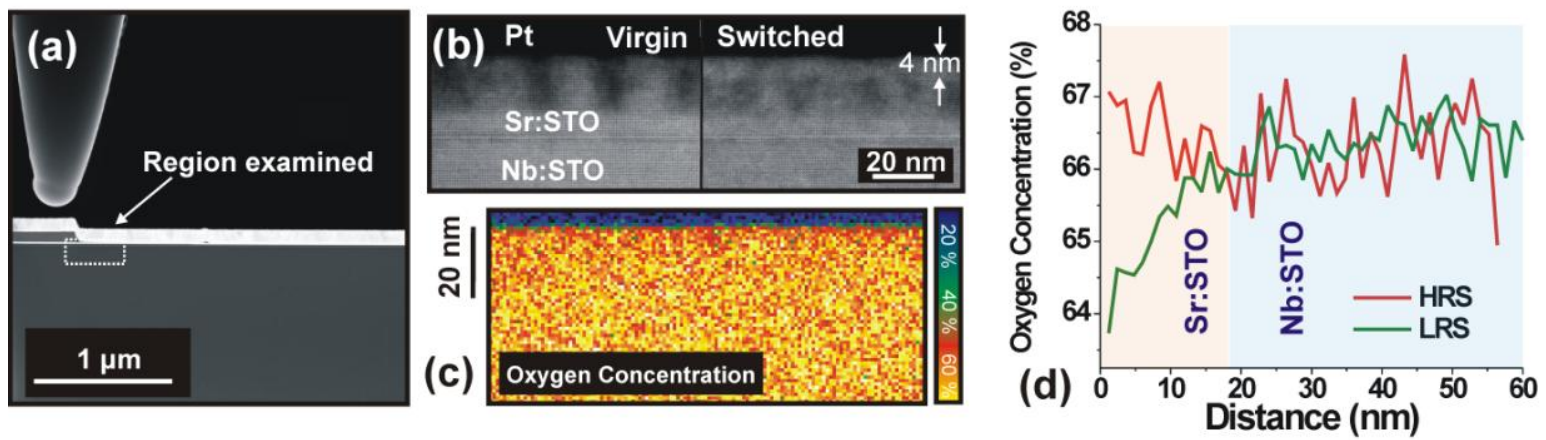

Figure 1. (a) STEM image of the experimental setup using the Nanofactory specimen holder.

BF STEM image showing the region of interest before and after forming. (c) Oxygen map obtained by EELS. (d) Oxygen concentration profiles acquired for the same device in a LRS and HRS.
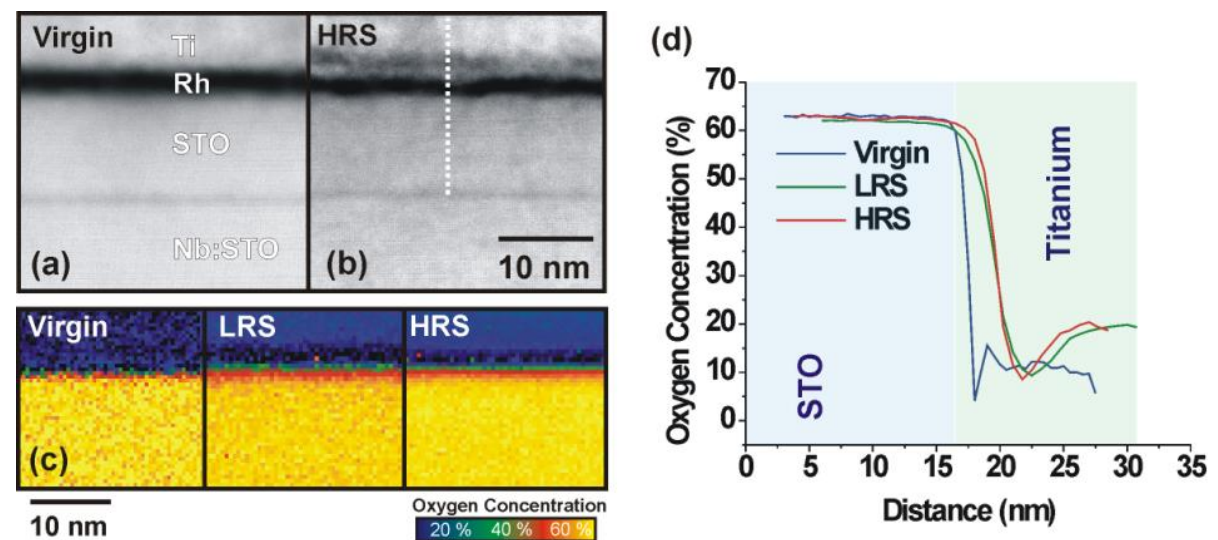

Figure 2. (a) and (b) STEM images of the $\mathrm{SrTiO}_{3}$ device before and after switching. (c) Oxygen maps of the same region acquired by EELS during switching. (d) Oxygen distribution in the devices during switching acquired from across the dotted line in (b). 